\title{
LA INNOVACIÓN EMPRESARIAL COMO IMPULSORA DE LAS PYMES EN LA REGIÓN CARIBE
}

Hugo Hernández-Palma Diego Cardona-Arbeláez Alex Raad-Pardo 
Panorama Económico, 24 (Octubre 2016 - Septiembre 2017), pp. 239-248

Hugo Hernández-Palma

Diego Cardona-Arbeláez

Alex Raad-Pardo

\title{
La innovación empresarial como impulsora de las Pymes en la Región Caribe
}

\section{Resumen}

En los últimos años, la pequeña y mediana empresa (pyme) ha venido jalonando el desarrollo sectorial en la Región Caribe. Como ente productivo, la pyme se ha convertido en un factor de competencia al interior del aparato económico nacional por su potencial para generar soluciones de empleo y producir ingresos constantes, a través de procesos comerciales, industriales y de servicios. Esto, la proyecta como una plataforma de posicionamiento económico de gran expectación. A nivel competitivo, la pyme tiene enormes ventajas inherentes que pueden derivar a largo plazo, en el fortalecimiento de los diversos sectores económicos que se vienen gestando en la región. A continuación, se analizará el componente de la innovación como un factor que ha impulsado el desarrollo y crecimiento de las pymes en la Región Caribe. A través de un ejercicio reflexivo del sector, se observarán conceptos que se vienen incorporando en los entornos empresariales a nivel regional y nacional.

Palabras Clave: Innovación, Pymes, Competitividad, Productividad y Región Caribe.

Clasificación JEL: D24, L11, O31, O32.

\section{Business innovation as a driver of Smes in the Caribbean Region}

\begin{abstract}
In recent years, small and medium-sized enterprises (SMEs) have been highlighting sectoral development in the Caribbean Region. As a productive entity, the SME has become a factor of competition within the national economic apparatus for its potential to generate employment solutions and produce constant income, through commercial, industrial and service processes. This, projected as a platform of economic positioning of great expectation. At the competitive level, the SME has enormous inherent advantages that can lead in the long term to the strengthening of the various economic sectors that are emerging in the region. Next, the innovation component will be analyzed as a factor that has driven the development and growth of SMEs in the Caribbean Region. Through a reflexive exercise of the sector, concepts will be observed that are being incorporated in the business environments at regional and national level.
\end{abstract}

Keywords: Innovation, SMEs, Competitiveness, Productivity and Caribbean Region.

JEL Classification: D24, L11, O31, O32.

\section{Innovation de l'activité de conduire des Pme dans la Region des Caraibes}

\begin{abstract}
Résumé
Récemment, les petites et moyennes entreprises (PME) ont surpassé le développement de la région caribéenne. Dans les trois secteurs d'activité (commercial, services et industriel), cette entité productrice (PME) est devenue un facteur compétitif au niveau national pour son potentiel générateur constant d'emplois et de revenus. Ceci crée beaucoup d'attentes en tant que plateforme économique. Au niveau compétitivité, cette entité a beaucoup d'avantages inhérents menant au renforcement à long terme de plusieurs secteurs économiques émergents de la région. Cet article analyse la composante innovation en tant que facteur promoteur de développement et croissance dans la région caribéenne. Après une réflexion sur le secteur, l'observation se fera sur des concepts incorporés chez les entrepreneurs régionaux et nationaux.
\end{abstract}

Mots-clés: Innovation, PME, compétitivité, productivité, Caraïbes.

Nomenclature JEL: D24, L11, O31, O32. 


\section{LA INNOVACIÓN EMPRESARIAL COMO IMPULSORA DE LAS PYMES EN LA REGIÓN CARIBE}

INFORMACIÓN DEL ARTÍCULO

Recepción de artículo: 9 de abril de 2016

Aceptación de artículo: 5 de agosto de 2016
Hugo Hernández-Palma

Universidad del Atlántico

Colombia

Diego Cardona-Arbeláez

Universidad Libre (Cartagena)

Colombia

Alex Raad-Pardo

Corporación Universitaria Larinoamericana

\section{INTRODUCCIÓN}

El propósito de esta iniciativa reflexiva, es explorar como la innovación entre los micro y pequeños empresarios puede jugar un papel fundamental en el despliegue de sus líneas de negocios. Por esta razón, se abordarán algunos conceptos y referencias asociadas al ámbito empresarial y el contexto nacional e internacional. Se mostrará cómo, a través de la innovación y proyección de las pymes, se ha venido consolidando en la Región Caribe un movimiento económico de gran alcance, que ha permitido la creación de pequeñas y medianas empresas en las principales ciudades y municipios de la costa atlántica. "Las pymes han alcanzado en las dos últimas décadas un crecimiento sostenido muy destacable y de gran impacto a nivel regional" (Gálvez, 2012), lo cual se justifica en gran medida por aspectos como el desarrollo tecnológico, proyección de comunidades productivas, políticas de estado, flexibilidad crediticia y la innovación. Según investigaciones que observan este fenómeno, la variedad de bienes y servicios, ha dado un perfil particular y se estima que muchos productos derivados de la innovación, han tenido gran acogida, lo que hace indispensable, pensar en cómo la puede replicarse en otros sectores productivos (Gálvez, 2012) (Caria \& Domínguez, 2015).

Asi mismo, se debe recordar que la innovación gira alrededor del talento humano, un stakeholder muy importante que las organizaciones no pueden excluir en el diseño de sus procesos. Antes bien, la empresa debe centrar sus esfuerzos en lograr que quienes la conforman tengan un papel esencial en la toma de decisiones, con un enfoque cada

${ }^{1}$ Autor para correspondencia. Correo electrónico: hugoghernandezpalma@gmail.com; diego.cardona@unilibrectg. edu.co; alexraad16@gmail.com 
vez más participativo, social y abierto, una característica fundamental de las organizaciones innovadoras (Del Rio, 2012; Cardona, 2011; Balza-Franco, 2015) (Castro, 2013) (Taylor, 2016).

Lo expuesto, hace necesario revisar en primera instancia, los conceptos teóricos de innovación con enfoque empresarial; seguidamente se mostrarán las reflexiones generadas a partir de este ejercicio y, por último, se contrastarán estos resultados con el propósito de realizar las conclusiones más importantes para el tema.

\section{CONSIDERACIONES SOBRE LA INNOVACIÓN EN LAS PYMES}

En años recientes, la innovación ha impulsado notablemente, el crecimiento económico de la Región Caribe y el entorno nacional. Sin embargo, advierte Romero (2013: pag.32), "lograr la inserción de este concepto en el panorama productivo no ha sido nada fácil y ha llevado tiempo y esfuerzo posicionar este componente en la mentalidad del empresario". Al analizar el concepto de innovación en los ambientes económicos, se pueden identificar dos tipos de líneas o direcciones para este importante eje temático: por un lado, como fuente de ingresos; por el otro, como mecanismo de sostenibilidad.

Según hipótesis planteadas por varios investigadores, en su función generadora de ingresos la innovación se enfoca en el trabajo creativo, que busca dar origen a nuevos productos o servicios orientados a satisfacer necesidades tangibles 0 intangibles, sin desconocer la rentabilidad que toda empresa debe arrojar como producto de su proceso operativo. Por otro lado, la innovación como fuente de sostenimiento se ocupa de todos los procesosquehacenqueuna empresa pueda solventar sus obligaciones e imposiciones y estar en constante dinamismo para estar a tono con las exigencias de un mercado cada vez más vertiginoso (Sáenz, Chang y Martínez, 2016).

Estos dos tipos de innovación se conocen en el mundo empresarial como agentes de cambio, los cuales ponderan las características competitivas de toda organización y, adicionalmente, contribuyen con la reinvención constante de los productos y servicios, lo que posibilita dar cumplimiento a los requerimientos de los diferentes mercados y sistemas. Al observar las ventajas de cada tipo de innovación, podemos evidenciar los aspectos más esenciales y, de esta manera, llevarlos al escenario real de manera más práctica. Ambos tipos de innovación tienen una serie de procesos inherentes que, al ejecutarse, permiten la rentabilidad, sostenibilidad, competitividad y vigencia para la pyme.

Autores como Amezquita y Baldovino (2005), han explicado que la innovación es un componente que genera valor en todos los sentidos, ya que a adoptarse como mecanismo de sostenimiento tanto el entorno como los actores del proceso desarrollan las dinámicas necesarias para renovar sus procesos, productos, bienes y/o servicios. En términos básicos, la innovación es un componente que sirve para la integración o asociación de toda una serie de procesos, elementos o variables, que de manera independiente no tendrían el impacto que pueden ofrecer cuando se aplican de manera conjunta o grupal.

Otros puntos de vista, han expresado que la innovación se debe relacionar con conceptos tan o más relevantes como creatividad, competitividad y sostenibilidad. Estos elementos son vitales cuando se 
quiere desarrollar una cadena de procesos que deriven en un cambio sustancial para generan un u nuevo escenario productividad, logrando así la ponderación de los resultados. Esto es clave cuando se quiere pasar, por ejemplo, de un mercado local a uno regional o de una nacional a uno internacional (Crissien, 2016).

En un estudio reciente Duarte y Bresan (2016), analizaron las respuestas de 211 participantes (propietarios, enólogos, directores y otros actores empresariales), con referencia a la innovación, encontrando aportes valiosos para entender, como ésta se inserta en las pymes. Entre los resultados más relevantes se tiene que, la innovación se asocia mayormente con la sostenibilidad y la gestión medioambiental o ecológica. Aspectos como la inversión, mejora de los procesos de almacenamiento, proyección de nuevos productos y análisis de las necesidades del consumidor, son los predominantes de atención. No obstante, lo anterior denota que aún existe muy poca investigación al respecto, por lo que se considera pertinente profundizar en las temáticas de esta área.

Por otra parte, investigadores como Birgit Pikkemaat y Anita Zehrer (2016), han expresado a través de sus estudios, los cuales han tenido por propósito explorar las cuestiones pertinentes a la experiencia de la innovación en empresas familiares, que las pequeñas células productivas, se enfrentan a serias falencias en cuanto a planeación estratégica e innovación. Normalmente, superar los obstáculos de manera frecuente, gracias a la solidaridad y apoyo de sus semejantes y afirman que el papel del cliente es fundamental para lograr renovaciones en productos y servicios. Lo anterior, deriva en la necesidad constante de recopilar información que se pueda tabular y analizar, para gestionar oportu- namente, los cambios requeridos en estas pequeñas unidades de negocio.

Siguiendo en la revisión de los avances en este sentido, se tiene que diversos autores han intentado describir el enfoque de innovación y como éste se utiliza para una pyme en el afán de generar soluciones en su actividad cotidiana. Al detallar el enfoque, se tiene que aspectos como un nombre de alto impacto para el negocio, asignación de funciones específicas para los cargos de relevancia y desarrollo de productos novedosos y diferenciales en su presentación, son a menudo las alternativas más frecuentes por las que optan muchas organizaciones. Este tipo de acciones, han permitido a estas pequeñas corporaciones, innovar de manera sistemática y pasar de un nivel muy pequeño a otro de mayor participación. Sin embargo, no siempre estas acciones son adoptadas por todos los emprendedores, y muchos de ellos incorporan de manera adicional componentes particulares según sus intereses, sin dejar a un lado las barreras que suponen la innovación para los pequeños emprendedores, por tal motivo la innovación debe enfocarse desde la concepción de la estrategia en las pequeñas empresas y trabajando colectivamente a partir de la asociatividad. (Mitra, 2000) (Taneja, S.; Golden Pryor y Hayek. 2016) (Kumar; Boesso; Favotto y Menini, 2012).

Estas pequeñas células productivas, son identificadas como motores de creatividad y por esta razón, muchos autores han expuesto que nunca se es tan pequeño como para quedar por fuera de un escenario de innovación. Con referencia a este aspecto, se conoce por ejemplo un estudio que muestra que, en Londres, tomando una muestra de 300 pequeños negocios se logró una caracterización del comportamiento empresarial, de estas unidades chicas. Se 
resalta que las empresas donde existe un alto nivel de motivación normalmente, logran crecer en ventas y/o empleados y a pesar de los obstáculos, logran subsistir de manera dinámica y competitiva en sus entornos. En este tipo de pymes, la innovación depende realmente de la capacidad de planeación que se logre desarrollar al interior del ente y así mismo el despliegue de otras ventajas competitivas se ve elevado (Moleiro y Teles, 2015) (Cifuentes, 2012).

\section{LA INNOVACIÓN EN LA EMPRESA}

Diferentes análisis, que han consultado a los pequeños empresarios sobre la concepción de innovación han expresado en su mayoría que la innovación es un aspecto esencial, ya que les permite mantenerse vigentes y con acceso a información de sumo interés para el desarrollo de nuevos productos y servicios (Corona, Montaño, y Ramírez, 2010). Estas acepciones, reiteran lo expuesto por varios autores latinoamericanos, quienes expresan que la innovación es una fuente dinamizadora de todo el universo empresarial y eleva los aspectos sustanciales para fomentar un crecimiento a futuro (Arriaga, López y Olivares, 2013) (Montoya, Montoya y Castellanos, 2010).

La investigación en el campo de la innovación deberá cobrar importancia, ya que de esta depende una mayor comprensión del fenómeno, y que aporte desde la academia hacia el sector real los factores claves de éxitos para la innovación y que a la vez sirva para nutrir la comunidad académica en sus esfuerzos investigativos (Romero, 2013).

\section{LOS PRINCIPALES BENEFICIOS DE LA INNOVACIÓN}

Sobre las ventajas de la innovación los líderes empresariales, consideran a la inno- vación algo favorable y sustancial para el desempeño organizacional; favoreciendo esta dinámica aspectos como la rentabilidad y maximización en el uso de los recursos (Araujo, A. (2010) (Romero, 2013).

Estaposicióntambiénguardaconcordancia con recientes teorías sobre innovación, las cuales sustentan que al innovar se debe promover que las variables asociadas al desempeño se vean mejoradas, teniendo en cuenta que la nueva posición involucra elementos comunes que potencian la calidad y las buenas prácticas en cualquier tipo de negocio (Ospina, Puche y Arango, 2014).

Además otro beneficio para las pequeñas y medianas empresas es que al apropiarse y mantener las practicas innovadoras, éstas deberán abrirse a trabajar en redes de colaboración lo que permite mayor sinergias y un mejor desempeño (Jong y Hulsink, 2012), que logra ser evidenciado a través de los datos recogidos en pequeñas empresas manufactureras en Cyprus, investigación realizada por (Dickson y Hadjimanolis 1998).

Asi mismo, los problemas sociales y los desafíos ambientales ofrecen oportunidades para los pequeños $\mathrm{y}$ medianos emprendedores para que desarrollen tecnologías limpias o verdes amigables con el medio ambiente $y$ puedan además encontrar en el camino de la sostenibilidad productos que sustituyan y que sean de impacto positivo para la sociedad y el medio ambiente (Carbal, 2011) (Vergara-Arrieta, 2015).

Finalmente, en el esfuerzo de las organizaciones por generar unas mejores prácticas empresariales con innovación y sentido social permiten generar un ambiente positivo al interior de la 
organización que son la base de todo esfuerzo para mejorar el desempeño de los empleados y por endedelas organizaciones (Fierro-Ulloa, 2012).

\section{LAS EXPECTATIVAS DEL PEQUEÑO EMPRESARIO FRENTE AL PROCESO DE INNOVACIÓN EMPRESARIAL}

Frente a este nuevo desafío, los empresarios esperan alcanzar mayor rentabilidad en su operación cotidiana, buscan proyectar los mercados y acceder a las novedades del mismo; esto con el propósito de lograr sostenibilidad y mantenerse vigente en un mundo tan cambiante. Esta última afirmación guarda trazabilidad con las nuevas que se están expandiendo a lo largo y ancho de Latinoamérica, y que hacen referencia a la innovación como un mecanismo de desarrollo económico integral que permite alcanzar tanto las metas financieras como aquellas relacionadas con el mercado y sus exigencias (Crissien, 2016) (Georgellis, Joyce y Woods, 2000).

\section{REFLEXIONES Y RECOMENDACIONES}

La innovación, como un mecanismo novedoso y moderno, viene siendo adoptada en el sector de las pymes, como una herramienta de proyección y promoción. Según las teorías consultadas, su aceptación se considera ya, un proceso práctico, lo cual propicia una buena receptividad en el sector empresarial. En la Región Caribe, este factor ha permitido potenciar la creación de pequeñas y medianas empresas. Con esto, no solo se generan nuevos puestos de trabajo, sino que se mejora la proyección de los sectores industriales y de servicios.

Para afianzar el proceso de expansión del enfoque innovador y los componentes asociados, las pequeñas unidades produc- tivas deben contemplar los siguientes elementos:

- Fomentar la capacitación constante del empresario y su equipo de trabajo; de esta manera se podría garantizar el acceso a información vigente $\mathrm{y}$ conocimiento de las nuevas teorías en los ámbitos de la economía, tanto nacional como internacional.

- Aprovechar todos los instrumentos que los entes gubernamentales, y no gubernamentales, ponen a disposición del sector empresarial para gestionar desde estos canales la apertura de nuevos mercados, clientes y proveedores.

- Facilitar las herramientas y recursos al interior de las pymes con el propósito de propiciar un escenario de aprendizaje y mejoramiento constante, que promueva en cada organización la renovación permanente de los ejes direccionales.

\section{REFERENCIAS BIBLIOGRÁFICAS}

Araujo, A. (2010). "La cultura organizacional innovadora: Desde una perspectiva valorativa". Revista Visión Gerencial. Vol. 9, No 2, Venezuela- Caracas. Pp. 219-239.

Arriaga, L.; López, C. y Olivares, E. (2013). Pymes: Contribuciones a la Economía y competitividad en México. Observatorio de la Economía, Vol. 6, 5-19.

Amezquita, J. y Baldovino E. (2005). Comportamiento innovador de las PYMES y grandes empresas en la industria química de Cartagena. Panorama Económico, Vol. 13, 133-151.

Balza-Franco \& Cardona (2015) "La Responsabilidad Social Empresarial y la lucha contra la pobreza" Revista Saber, ciencia y libertad. Pág. 115-124. Vol. 10 No 1. Recuperado de: https://dialnet.unirioja.es/servlet/articulo?codigo $=5329117$.

Carbal-Herrera, A. (2011). Una aproximación cognoscitiva sobre la medición y valoración 
del medio ambiente bajo el paradigma contemporáneo. Saber, ciencia y libertad, 6(2), 121-129.

Cardona-Arbeláez, D. \& Hernandez (2011) "La perspectiva de los gerentes de las pymes hoteleras frente a la Responsabilidad Social Empresarial en la ciudad de Cartagena". Revista Saber, ciencia y libertad. Pág. 91-104. Vol 6 No 1. Recuperado de: https:// dialnet.unirioja.es/servlet/articulo?codigo $=3997354$.

Caria, S. Domínguez, R. (2015). Estructura productiva primario-exportadora y Buen Vivir en Ecuador, 2009-2014. Revista Internacional de Cooperación y Desarrollo, 2(1), 37-64. Doi: http://dx.doi. org/10.21500/23825014.2232

Castro, A. (2013). Ética empresarial en la responsabilidad social universitaria. Aglala, Vol. 4, 1,1-20.

Cifuentes, O. (2012). Aplicación del alineamiento del Supply Chain Management como estrategia competitiva en las empresas del Clúster Logístico de Cartagena. Revista Aglala Vol. 3, 1, 122-146.

Corona, J. R., Montaño, O. y Ramírez, I. (2010). Innovación en la Pymes: Desarrollo de un modelo para el estado de Hidalgo. ACACIA (Ed), XIV Congreso Anual de la Academia de Ciencias Administrativas, Cap. Innovación y Tecnología. Mty., NL. Mex.

Crissien, J. (2016). Espíritu empresarial como estrategia de competitividad y desarrollo económico. Revista EAN, Vol. 57, 104-115.

Del Río-Cortina, J. (2012). Desarrollo de actividades de emprendimiento con innovación y responsabilidad social en los hoteles en la ciudad de Cartagena de Indias. Saber, Ciencia y Libertad, 7 (2), 113-124. Recuperado de: https://dialnet.unirioja.es/servlet/articulo?codigo $=5109395$.

Dickson, K.E; Hadjimanolis, A. (1998) "Innovation and networking amongst small manufacturing firms in Cyprus", International Journal of Entrepreneurial Behavior \& Research, Vol. 4 Issue: 1, pp.5-17, doi: 10.1108/13552559810203939.
Duarte, A. y Bressan, A. (2016). Micro and small business innovation in a traditional industry, International Journal of Innovation Science, Vol. 8 Issue: 4, pp.311-330, doi: 10.1108/IJIS-06-2016-0013.

Fierro-Ulloa, I. (2013). Comportamiento organizacional positivo: implicaciones para la organización actual. Saber, Ciencia y Libertad, 8 (2), 103-111. Recuperado de: https:// dialnet.unirioja.es/servlet/articulo?codigo $=5104984$.

Georgellis, Y., Joyce, P. y Woods, A. (2000). Entrepreneurial action, innovation and business performance: the small independent business, Journal of Small Business and Enterprise Development, Vol. 7 Issue: 1, pp.717, doi: 10.1108/EUM0000000006801.

Gálvez, E. (2012). Impacto de la innovación sobre el rendimiento de la mí pyme: un estudio empírico en Colombia. Estudios Gerenciales, Vol. 28 n. 122, 11-27.

González, M., Rangel, J. y Aguilera, L. (2015). La influencia de la estrategia empresarial en la innovación y las finanzas de la pyme manufacturera de Aguascalientes. Red Internacional de Investigadores de Competitividad, vol. 9, 861-873.

Jong, Jeroen; Hulsink, Willem (2012) "Patterns of innovating networking in small firms", European Journal of Innovation Management, Vol. 15 Issue: 3, pp.280-297, doi: 10.1108/14601061211243639.

Kumar, Kamalesh, Boesso, Giacomo; Favotto, Franceso; Menini, Andrea (2012) "Strategic orientation, innovation patterns and performances of SMEs and large companies", Journal of Small Business and Enterprise Development, Vol. 19 Issue: 1, pp.132-145, doi: 10.1108/14626001211196442.

Mitra, Jay (2000) "Making connections: innovation and collective learning in small businesses", Education + Training, Vol. 42 Issue: 4/5, pp.228-237, doi: 10.1108/00400910010373679.

Moleiro, J. y Teles, M. (2015). Too small to innovate? Creating value with fewer resources, Journal of Business Strategy, Vol. 36 Issue: 2, pp.25-33, doi: 10.1108/JBS-02-2014-0014. 
Montoya R., A.; Montoya R., I. y Castellanos, O. (2010). Situación de la competitividad de las Pyme en Colombia: elementos actuales y retos. Agronomía Colombiana, 28 (1), 107-117.

Ospina, M.; Puche, M. y Arango, B. (2014). Gestión de la Innovación en Pequeñas y Medianas Empresas. Generando ventajas competitivas y posicionamiento en el Mercado. Gestión de las personas y tecnología, 19, 34-39.

Pikkemaat, B. y Zehrer, A. (2016). Innovation and service experiences in small tourism family firms, International Journal of Culture, Tourism and Hospitality Research, Vol. 10 Issue: 4, pp.343-360, doi: 10.1108/ IJCTHR-06-2016-0064.

Romero, L. (2013). Competitividad y Productividad en empresas familiares pymes: una aproximación desde la interacción familia-empresa. Revista EAN, Vol. 57, 131-142.

Romero, Z. (2013). Hacia un nuevo modelo de medición que refleje las capacidades investigativas de los colombianos. Saber Ciencia Y Libertad, Vol. 8(2), 13-16. doi:http://dx.doi. org/10.22525/sabcliber2013v8n2.100.

Sáenz, D.; Chang, E. y Martínez, J. (2016). El impacto de la cultura organizacional sobre la estrategia competitiva y su influencia en el desempeño exportador de las pymes de Barranquilla, Colombia. TEC Empresarial, Vol. 10, No. 2, 10-15.

Taneja, Sonia; Golden Pryor M, Hayek, M (2016) "Leaping innovation barriers to small business longevity", Journal of Business Strategy, Vol. 37 Issue: 3, pp.44-51, doi: 10.1108/ JBS-12-2014-0145.

Taylor, R. (2016). Intraemprendimiento incluyente, modelo de sostenibilidad de empleo y cooperación en el Centro de Emprendimiento Pedro Romero. Revista Internacional de Cooperación y Desarrollo, 3(2), 35-55. Doi: http://dx.doi.org/10.21500/23825014.2541

Vergara Arrieta, J., \& Carbal Herrera, A. (2015). Diseño de un sistema de gestión en responsabilidad social empresarial para pequeños hoteles de la ciudad de Cartagena. Saber Ciencia Y Libertad, 9(2), 91108. doi:http://dx.doi.org/10.22525/sabcliber.2014v9n2.91108.

Hernández-Palma, H., Cardona-Arbeláez, D., \& Raad-Pardo, A. (2016). La innovación empresarial como impulsora de las Pymes en la Región Caribe. Panorama Económico, 24, 239-248.

\section{AUTORES}

Hugo Hernández-Palma

Magíster en Sistemas de Gestión de la Universidad autónoma del Caribe. Especialista estudios pedagógicos Corporación Universitaria de la Costa y Especialista en diseño y evaluación de proyectos de la Universidad del Norte. Ingeniero Industrial Universidad Libre Barranquilla. Docente universitario del programa Administración de Empresas dela Universidad del Atlántico

Diego Cardona-Arbeláez

Doctorante de Administración de la Universidad del Norte, Magister en desarrollo empresarial de la Universidad del Magadalena, especialista en Gerencia de Mercadeo de la Universidad Jorge Tadeo Lozano. También es especialista en Docencia Universitaria. Administrador de Empresas. Docente investigador de la Universidad Libre de Cartagena

\section{Alex Raad-Pardo}

Especialista en Finanzas y Negocios Internacionales, Contador Público, Consultor Contable y Financierc 


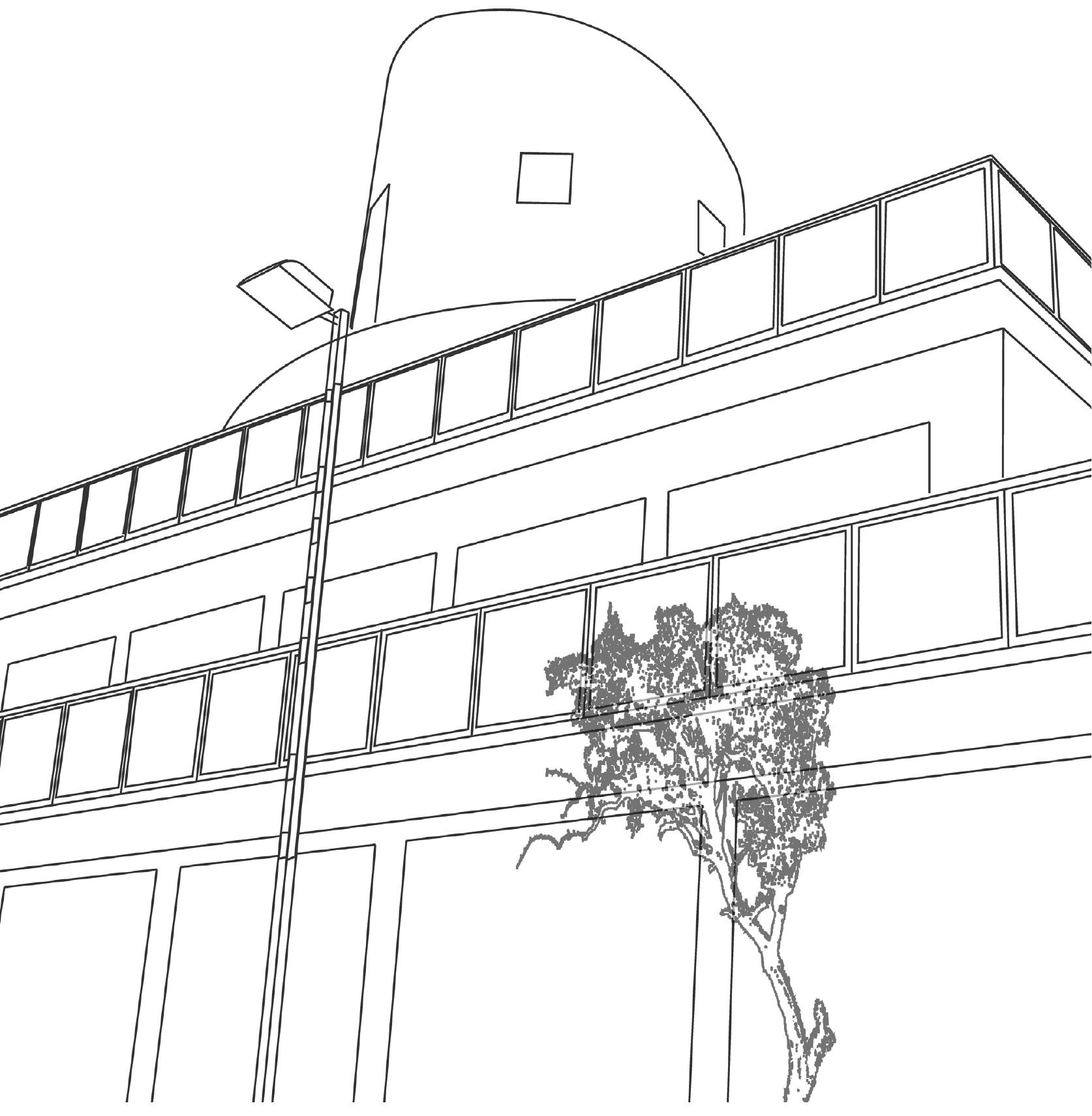

